09

\title{
Эффективность магнитооптического преобразования магнитных потоков, создаваемых матрицей высокотемпературных сверхпроводников
}

\author{
(C) E.A. Протасов \\ Национальный исследовательский ядерный университет „МИФИ“, \\ 115409 Москва, Россия \\ e-mail: protasovea@mail.ru
}

(Поступило в Редакцию 24 мая 2017 г.)

Рассмотрена возможность использования одноосных феррит-гранатовых магнитооптических пленок с полосовой доменной структурой для визуализации пространственного распределения магнитного потока вблизи чувствительных элементов матрицы высокотемпературных сверхпроводников. Предложены способы, повышающие эффективность магнитооптического преобразования.

DOI: $10.21883 /$ JTF.2018.08.46314.2353

\section{Введение}

В работе [1] была предложена концепция построения нового типа приемника тепловых излучений, основанная на уникальных магнитных свойствах матрицы высокотемпературных сверхпроводников (ВТСП) и магнитооптическом способе считывания с нее информации. Главными элементами такого устройства являются матрица ВТСП, содержащая до $\sim 10^{5}$ чувствительных элементов (ЧЭ), и магнитооптический преобразователь (МОП), использующий эффект Фарадея в эпитаксиальных феррит-гранатовых пленках (ЭПФГ).

Идеальными с точки зрения практического использования в устройствах визуализации магнитных полей являются феррит-гранатовые магнитооптические (МО) пленки с максимальным значением угла фарадеевского вращения $\theta_{F}$, малым оптическим поглощением, отсутствием или малым значением коэрцитивности, линейной кривой намагничивания, малыми размерами доменов или их отсутствием. Однако не всегда удается в одном образце сочетать указанные свойства оптимальным образом. Так, например, наибольшим значением $\theta_{F}$, достигающим нескольких десятков тысяч градусов на сантиметр, обладают феррит-гранатовые пленки, содержащие висмут. При длине волны $\lambda=0.375 \mu \mathrm{m}$ угол фарадеевского вращения в одноосных висмутсодержащих пленках может достигать $60 \mathrm{grad} / \mu \mathrm{m}$. Но наличие полосовой доменной структуры, присущее таким пленкам, ограничивают их разрешающую способность.

Пленки, не содержащие висмут с анизотропией „легкая плоскость“, обладают практически неограниченной разрешающей способностью, однако требуют для своего намагничивания значительных магнитных полей.

В работе [1] использовались преимущественно пленки феррит-гранатов с анизотропией легкая плоскость. Однако малая статическая восприимчивость ограничивает возможности применения пленок с плоскостной анизотропией в тех случаях, когда магнитные поля, создаваемые исследуемыми структурами, достаточно малы, не превышают десятка эрстед. Именно с такими полями приходится работать в условиях, когда температура ВТСП структуры приближается к критической температуре $T_{\mathrm{cr}}$, что продиктовано необходимостью получения максимальной чувствительности приемника тепловых изображений. В то же время при использовании матричной ВТСП структуры для приема ИК излучения с длинами волн $\sim 10-20 \mu \mathrm{m}$ требования на разрешающую способность существенно снижаются и в этих условиях вполне приемлемыми могут оказаться висмутсодержащие пленки с одноосной анизотропией, которые, как отмечалось, обладают наибольшими значениями магнитооптических параметров в малых внешних магнитных полях.

Основной задачей настоящей работы является исследование возможности использования одноосных ферритгранатовых пленок с полосовой доменной структурой с целью увеличения эффективности преобразования магнитных потоков, создаваемых ВТСП матрицей.

\section{Методика измерений}

При изучении процессов намагничивания ВТСП пленок измерения магнитных характеристик ЭПФГ и визуального наблюдения пространственного распределения магнитного поля в матричной ВТСП структуре была создана экспериментальная установка, представленная на рис. 1. Установка состоит из двух частей: системы визуального наблюдения и измерительной системы. Первая состоит из осветителя, объектив которого формировал параллельный пучок белого света, который проходил через делительный кубик 2 и поляризатор 3 и попадал на полупрозрачное зеркало 4. Отражаясь от этого зеркала, свет проходил через окно 5 азотного криостата 6, где попадал на узел преобразования 7 , состоящий из матрицы ВТСП и МО пленки с зеркальным покрытием. Далее пучок отражался от зеркального покрытия МО пленки, 


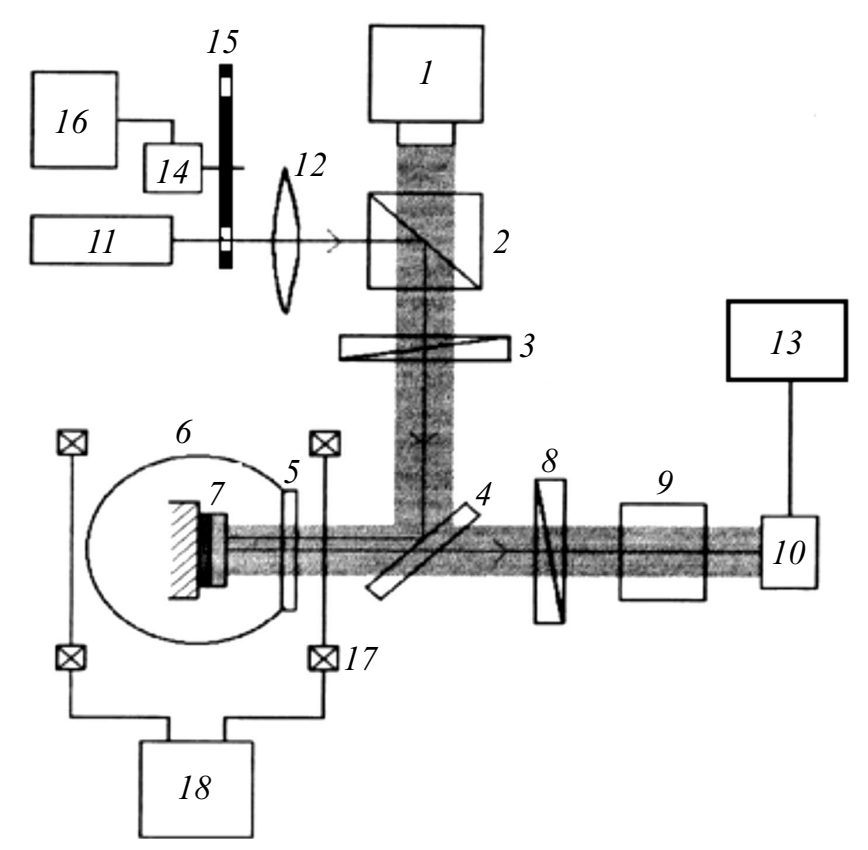

Рис. 1. Схема измерительной установки: 1 - осветитель, 2 - делительный кубик, 3 - поляризатор, 4 - полупрозрачное зеркало, 5 - прозрачное окно, 6 - азотный криостат, 7 - МО пленка + матрица ВТСП, 8 - анализатор, 9 - объектив, 10 - фотодиод или цифровой фотоаппарат, 11 - лазер, 12 - фокусирующая линза, 13 - селективный микровольтметр В6-9, 14 - электродвигатель, 15 - диск с отверстиями, 16 - генератор Г3-33, 17 - катушки Гельмгольца, 18 - блок питания ТЕС-5020.

последовательно проходил через полупрозрачное зеркало 4 , анализатор 8 и попадал в объектив 9, который формировал изображение на чувствительной матрице цифрового фотоаппарата 10. Измерительная схема была построена следующим образом. Промодулированный луч $\mathrm{Ne}-$ Не-лазера мощностью $5 \mathrm{~mW} 11$ фокусировался линзой 12 и поступал на делительный кубик 2. Далее луч проходил по тому же пути, что и пучок белого света, с тем отличием, что в момент измерений цифровой фотоаппарат был заменен на фотодиод ФД-20КП, сигнал с которого поступал на вход селективного микровольтметра В6-9 13. В качестве модулятора использовалась система, состоящая из электродвигателя 14 и диска с отверстиями 15. Электродвигатель питался от генератора ГЗ-33 16. Частота модуляции составляла $f=33 \mathrm{~Hz}$.

Криостат был установлен между катушками Гельмгольца 17 , которые позволяли формировать однородное магнитное поле, перпендикулярное поверхности ВТСП. Катушки питались от блока питания ТЕС-5020 18. Максимальный ток, подаваемый на катушки, составлял $4.5 \mathrm{~A}$, что при эффективности катушек 40 Ое/А позволяло получать магнитные поля напряженностью до $H=180$ Oе. Такая конструкция установки позволила непосредственно наблюдать целиком структуру сверхпроводника и направлять лазерный луч в ту область поверхности пленки ВТСП, где необходимо провести измерения.
Установка обладала максимальной разрешающей способностью $300 \mathrm{~cm}^{-1}$ и чувствительностью к магнитному полю в режиме визуализатора $10^{-4}$ и $10^{-5} \mathrm{~T}-$ в режиме регистрации сигнала. Следует отметить, что качество прижима пленки ВТСП к ЭПФГ существенно влияет на контраст и разрешение отдельных деталей, а также на величину измеряемого поля визуализируемого объекта. Наличие неконтролируемого зазора между ВТСП и пленкой ЭПФГ величиной несколько микрон приводит, даже в случае линейного спада напряженности локального поля у края сверхпроводника, к многократному уменьшению его влияния на величину намагниченности магнитооптической пленки.

Оптимальная толщина $h_{\text {opt }}$ пленки, при которой достигается наибольшая эффективность МО преобразования

$$
h_{\mathrm{opt}}=\left(2 \cdot \theta_{\mathrm{F}}\right)^{-1} \operatorname{arctg}\left(4 \cdot \theta_{\mathrm{F}} / \alpha\right) \text {, }
$$

где $\theta_{\mathrm{F}}$ - удельное фарадеевское вращение, $\alpha-$ коэффициент поглощения материала. Значение $\theta_{\mathrm{F}}$ в области $\lambda=0.63 \mu \mathrm{m}$, измеренное нами на пленке состава $\mathrm{Bi}_{1.3}\left(\mathrm{Y}_{2} \mathrm{La}_{1.7}\right) \mathrm{Fe}_{5} \mathrm{O}_{12}$, достигало $5.5 \mathrm{grad} / \mu \mathrm{m}$ при $T=295 \mathrm{~K}$ и оптимальная толщина пленки в соответствии с (1) должна быть $h_{\mathrm{opt}}=0.5 \mu \mathrm{m}$.

Толщина пленки ограничивается минимальным пространственным периодом визуализируемого магнитного поля $b$, так как поле рассеяния $\left(H_{\mathrm{disp}}\right)$ спадает по экспоненциальному закону с увеличением расстояния от поверхности $\left(H_{\text {surf }}\right)$ источника поля

$$
H_{\text {disp }}=H_{\text {surf }} \exp (-2 \pi h / b) .
$$

Здесь $h$ - толщина пленки. (Толщина МО пленок, используемых в настоящей работе, варьировала от 0.1 до $2 \mu \mathrm{m}$.)

\section{Исследование преобразования магнитных потоков, создаваемых ВТСП матрицей, и измерение параметров пленок ЭПФГ с различной анизотропией}

На рис. 2 представлены кривые намагничивания ЭПФГ с одноосной анизотропией и анизотропией „легкая плоскость“. По оси ординат откладывалось значение сигнала с фотоприемника, из которого вычиталось минимальное значение, и затем извлекался корень. Это связано с тем, что интенсивность сигнала в эффекте Фарадея пропорциональна квадрату синуса угла поворота плоскости поляризации $\beta$, который, в свою очередь, пропорционален величине магнитного поля $H_{S}$, прикладываемого к образцу $\left(\beta=V H_{S} h\right.$, где $V$ - постоянная Верде). Для $\mathrm{Bi}_{1.3}\left(\mathrm{Y}_{,} \mathrm{La}_{1.7}\right) \mathrm{Fe}_{5} \mathrm{O}_{12}$ на длине волны $\lambda=0.8 \mu \mathrm{m}, V=7.2 \mathrm{grad} / \mathrm{A}$ :

$$
I \sim I_{0}\left[(1-p) \sin ^{2}(\beta+\Phi)+p\right] .
$$




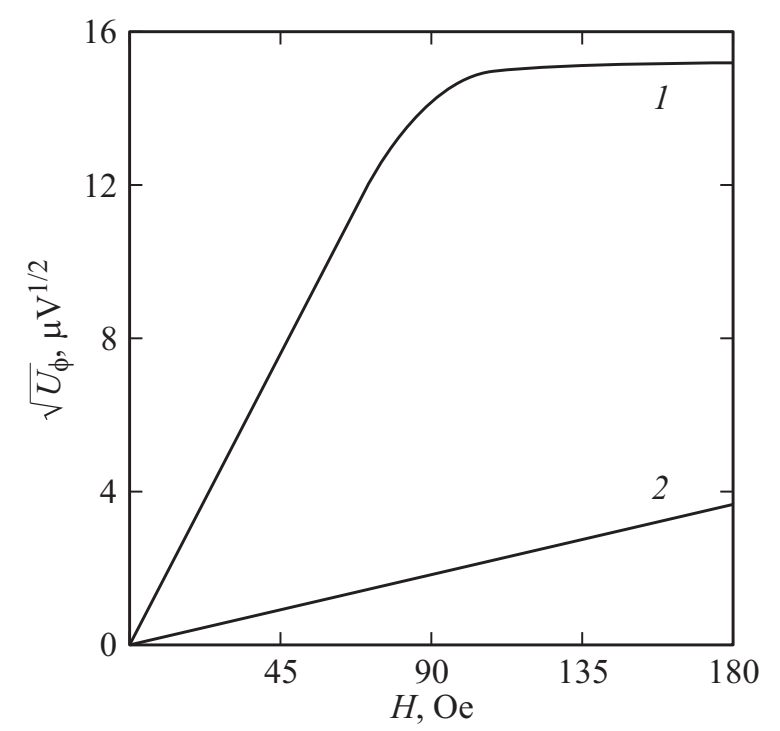

Рис. 2. Кривые намагничивания ЭПФГ с анизотропией: 1 - „одноосная“, 2 - ,легкая“ плоскости.

Здесь $I$ и $I_{0}-$ интенсивности прошедшего и падающего излучений соответственно, $p$ - коэффициент, учитывающий деполяризацию, а

$$
\Phi=\theta_{\mathrm{F}}\left(M / M_{s}\right) h
$$

где $M$ и $M_{s}$ - магнитный момент единицы объема и намагниченность насыщения.

В идеале поляризаторы должны полностью запирать луч лазера, однако из-за наличия деполяризации на оптических элементах системы всегда присутствует некоторый постоянный уровень сигнала. Вычитание этого сигнала позволяет тем самым учесть влияние деполяризации.

В результате имеет место линейная зависимость для пленки с анизотропией ,легкая плоскость“ во всем диапазоне магнитных полей, а для „одноосной“ пленки наблюдается насыщение в области $H \sim 90$ Ое.

Из сравнения кривых намагничивания видно, что чувствительность одноосной магнитооптической пленки существенно превышает чувствительность ЭПФГ с анизотропией „легкая плоскость“, особенно в малых магнитных полях.

Были измерены поля коэрцитивности нескольких МО образцов с одноосной анизотропией, используемых в работе. Лучшие образцы МО пленок обладали полями коэрцитивности $H_{c}=(0.1-0.3)$ Ое. Такие низкие значения $H_{c}$ достигаются, если применять жесткие требования к обработке и ориентации подложек и осуществлять специальные меры по термоотжигу выращенных пленок. Характерные поля насыщения для образцов с одноосной анизотропией $H_{S}=(50-100)$ Oе.

Отношение полей насыщения к полям коэрцитивной силы определяют динамический диапазон реагирования на внешние магнитные поля, который составляет для подобных пленок $10^{2}-10^{3}$.
Рассмотрим особенности преобразования магнитных потоков ВТСП матрицей, состоящей из большого количества тонкопленочных (толщина $0.2 \mu \mathrm{m}$ ) чувствительных элементов (4096 шт.) на основе $\mathrm{Y}_{1} \mathrm{Ba}_{2} \mathrm{Cu}_{3} \mathrm{O}_{7-\delta}$, которая в отсутствие магнитного поля охлаждается до температуры ниже критической, и лишь после этого включается внешнее магнитное поле напряженностью $H_{0}$. Поскольку в этом случае магнитный поток не проникает в сверхпроводящие элементы матрицы (имеет место так называемый режим экранирования), он существенно трансформируется и представляет собой пространственно-периодическую структуру с периодом, равным расположению ЧЭ. Плотность магнитного потока В в элементах матрицы равна нулю и максимальна в промежутках между ЧЭ. Если вплотную к сверхпроводниковой матрице расположить МО пленку, то структура поля отобразится в виде соответствующей структуры намагниченности МО пленки, которая, в свою очередь, может быть визуализирована. Характерная фотография визуализированного распределения магнитного потока, создаваемого ВТСП матрицей с помощью ЭПФГ с плоскостной анизотропией в магнитном поле напряженностью $130 \mathrm{Oe}$, изображена на рис. 3.

Однако для получения приемлемого по контрасту изображения необходимо к таким пленкам прикладывать магнитные поля напряженностью несколько десятков или даже сотен эрстед. При температурах, близких к критической температуре $T_{\mathrm{cr}}$, магнитные поля такой напряженности могут приводить к частичной потере сверхпроводимости ЧЭ, что нежелательно.

Известно [2], что максимальная тепловая чувствительность ЧЭ достигается при рабочей температуpe, отличающейся от $T_{\text {cr }}$ всего на $0.5 \mathrm{~K}$. При этом напряженность внешнего магнитного поля для пленок $\mathrm{Y}_{1} \mathrm{Ba}_{2} \mathrm{Cu}_{3} \mathrm{O}_{7-\delta}$ не должна превышать несколь-

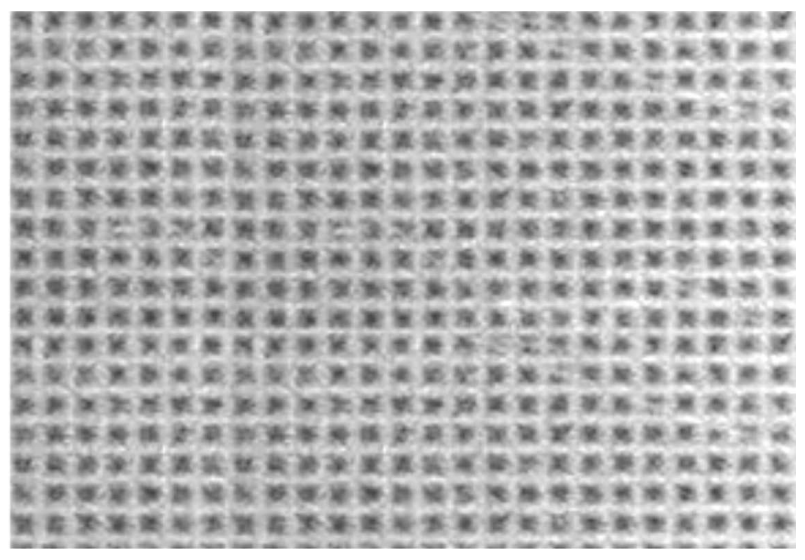

Рис. 3. Фото визуализированного изображения структуры магнитного поля вблизи ВТСП матрицы, полученного с помощью МО пленки с анизотропией „легкая плоскость“ в магнитном поле 130 Ое. (Наблюдаемые царапины возникли вследствие несовершенства подложки.) 
ких единиц эрстед, что накладывает ограничения на тип используемой для визуализации феррит-гранатовой пленки.

Рассмотрим подробнее структуру магнитных полей, возникающих вокруг элементарной сверхпроводящей ячейки. Размер ячейки определяется светосилой (телесным углом) инфракрасной оптики, формирующей тепловое изображение на ВТСП матрице, максимальной длиной волны теплового излучения, критерием выбора убывания амплитуды фокального пятна и характера расходимости регистрируемого излучения. Если объект находится на расстоянии, значительно превышающем фокусное расстояние ИК объектива $-f$, то тепловую волну можно считать плоской. Если при этом размер ЧЭ ограничить площадью, занимаемой первым дифракционным максимумом, то площадь ЧЭ

$$
S_{s e}=1.5 \lambda^{2} / \Omega
$$

Для длин волн $\lambda=20 \mu \mathrm{m} \quad$ и $\Omega=0.1 \mathrm{sr} \quad$ (светосила $1: 3$ ) площадь чувствительного элемента $S_{s e}=6 \cdot 10^{-5} \mathrm{~cm}^{2}$. При этом размер ячейки квадратной формы $a=90 \mu \mathrm{m}$.

Нами была изготовлена ВТСП матрица, имеющая $64 \times 64$ чувствительных элементов размером $100 \times 100 \mu \mathrm{m}$, расположенных с периодом $b=150 \mu \mathrm{m}$.

На рис. 4 изображен фрагмент такой матрицы и представлено рассчитанное распределение поля вокруг одной из ячеек для нормальной

$H_{z} / H_{0}=\frac{1}{\pi}\left(\operatorname{arctg} \frac{x-(b-a) / 2}{\Delta}-\operatorname{arctg} \frac{x+(b-a) / 2}{\Delta}\right)$

и тангенциальной

$$
H_{\perp} / H_{0}=\frac{1}{2 \pi} \ln \frac{(x+(b-a) / 2)^{2}+\Delta^{2}}{(x-(b-a) / 2)^{2}+\Delta^{2}}
$$

компонент напряженности магнитного поля. Этот расчет (рис. $4, b, c)$ проведен для двух отличающихся в два раза расстояний от поверхности сверхпроводниковой пленки до магнитооптической $-\Delta$.

\section{Обсуждение результатов}

Как видно из рис. 4, в режиме экранирования магнитный поток концентрируется в промежутках между ЧЭ и напряженность его нормальной компоненты $H_{n}$ :

$$
H_{n}=\left[\left(S_{n}+S_{s e}\right) / S_{n}\right] H_{0},
$$

где $S_{n}$ - площадь промежутка между чувствительными элементами, $H_{0}$ - напряженность внешнего магнитного поля. В нашем случае $H_{n}=2.0 \cdot H_{0}$. Искривление магнитного потока приводит к появлению тангенциальной составляющей $H_{\perp}$, максимум которой находится вблизи края ЧЭ и по величине равен $H_{n}$. Следует особо отметить, что если при нарушении сверхпроводимости (при температуре выше $T_{\mathrm{cr}}$ ) $H_{n}$ становится равным $H_{0}$,

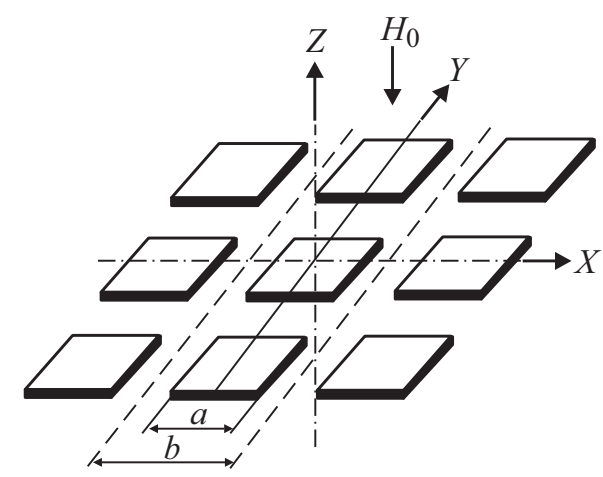

$a$

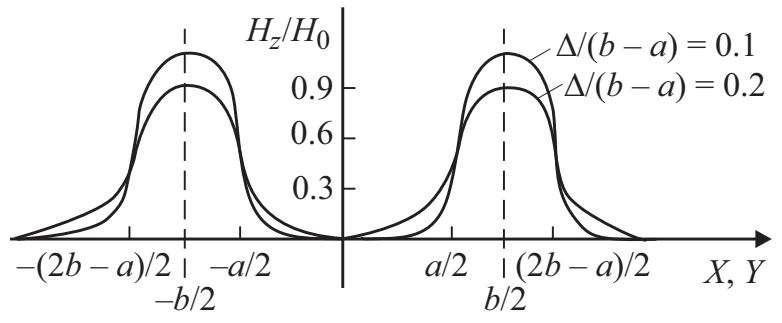

$b$

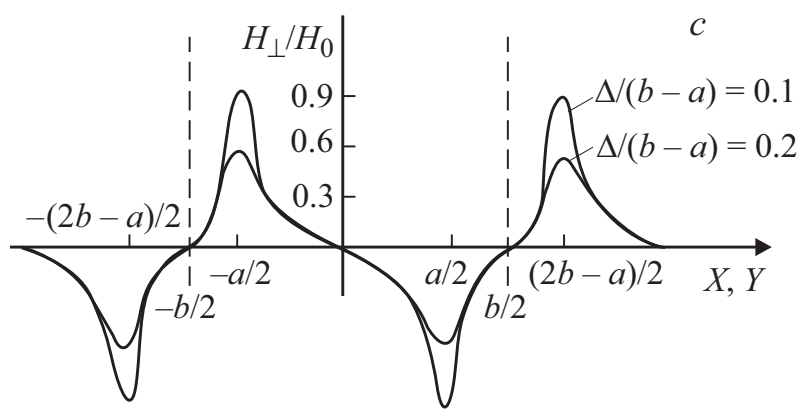

Рис. 4. Фрагмент матрицы из ВТСП чувствительных элементов $(a)$. Распределение нормальной $(b)$ и тангенциальной $(c)$ компонент напряженности магнитного поля. Здесь $\Delta-$ расстояние до точки регистрации.

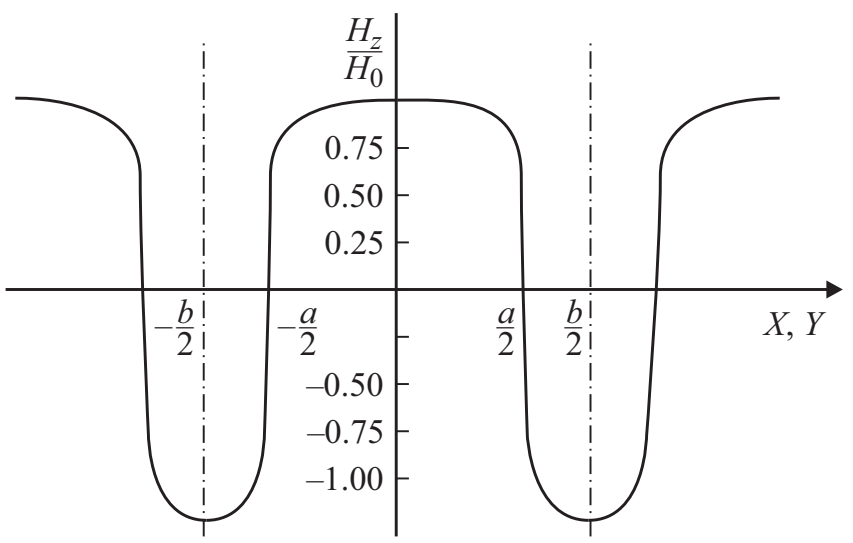

Рис. 5. Распределение нормальной компоненты магнитного потока, захваченного ВТСП элементами матрицы при охлаждении в магнитном поле напряженностью $H_{0}$ для $\Delta /(b-a)=0.1$. 


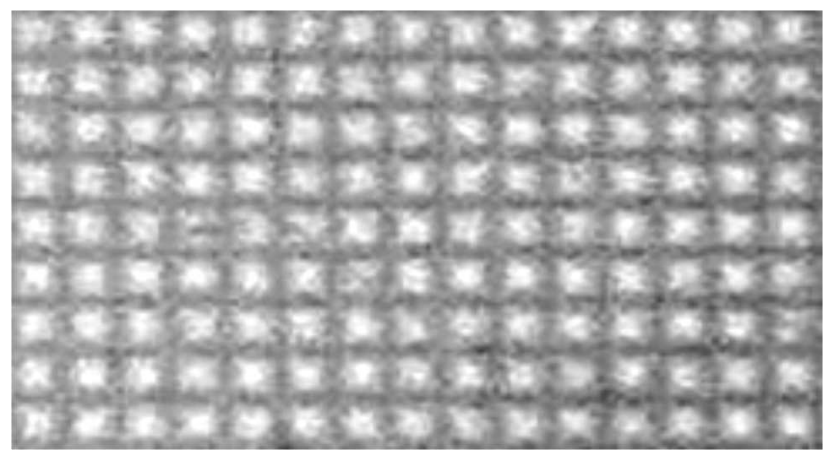

Рис. 6. Визуализированное с помощью феррит-гранатовой пленки с „одноосной“ анизотропией изображение фрагмента матрицы с захваченным магнитным потоком. Величина захваченного магнитного потока одного элемента матрицы составляет $58 \mathrm{G}$.

т. е. уменьшается всего в два раза, то $H_{\perp}$ в этом случае уменьшается практически до нуля. Остаточное поле $H_{\perp}$ может появиться в результате случайного наклона оси магнитной системы по отношению к нормали ВТСП матрицы и малых возмущений внешнего магнитного потока соседними ЧЭ. В любом случае остаточное $H_{\perp}$ не превышает нескольких процентов от $H_{0}$. Из приведенного расчета видно, что амплитуда сигнала довольно существенно уменьшается при увеличении расстояния между ЧЭ и МО пленкой.

Другое практически важное состояние намагниченности возникает при охлаждении ВТСП структуры во внешнем магнитном поле, которое выключается при достижении температуры ниже $T_{\mathrm{cr}}$ [3]. Матрица охлаждалась до температуры $76 \mathrm{~K}$ в магнитном поле 130 Ое. Отметим, что критическая температура $T_{\text {cr }}$ используемой матрицы ВТСП составляла $87 \mathrm{~K}$.

Благодаря значительной силе пиннинга происходит захват магнитного потока элементами матрицы. При выключении внешнего магнитного поля ВТСП ячейка оказывается намагниченной. Величина захваченного магнитного потока одним элементом матрицы ВТСП составила $58 \mathrm{G}$. Поля рассеяния над ячейкой и в промежутках оказываются направленными в противоположные стороны (рис. 5). Это обстоятельство позволяет использовать для визуализации магнитных распределений одноосные феррит-гранатовые пленки, обладающие, как отмечалось выше, большими углами фарадеевского вращения. Характерное изображение матрицы ВТСП с захваченным магнитным потоком представлено на рис. 6.

Как известно [4], в размагниченном состоянии в одноосном магнитном кристалле существует доменная структура (ДС). При распространении света в магнитной среде происходит фазовый сдвиг между право- и левополяризованными волнами. Знак и величина сдвига определяются векторным значением проекции магнитного момента на направление распространения света. Таким образом, кристалл с ДС является фазовой решеткой для световой волны, поляризованной по кругу. То же относится и к линейно поляризованному свету.

Исследования [4] показали возможность значительного изменения параметров ДС с помощью магнитного поля, теплового, механического воздействия на кристалл, т.е. магнитная решетка является перестраиваемой. В тонких пленках толщиной $h=1-10 \mu \mathrm{m}$ период ДС также равен $1-10 \mu \mathrm{m}$. Анализ размеров доменов одноосной плоскопараллельной пленки с поверхностью, перпендикулярной оси анизотропии, показывает, что с увеличением толщины МO пленки размер домена растет, как корень квадратный из толщины пленки. Нужно отметить, что этот анализ базируется на предположении о плоскопараллельной форме доменов. Такие структуры как раз наблюдаются в тонких пленках. Таким образом, с помощью тонких одноосных МО пленок можно получить разрешающую способность, достаточную для визуализации матричной ВТСП структуры, имеющей период $\sim 100 \mu \mathrm{m}$.

\section{Заключение}

Проведенный в настоящей работе анализ возможностей использования одноосных феррит-гранатовых пленок с полосовой доменной структурой для визуализации пространственного распределения магнитного потока вблизи чувствительных элементов матрицы ВТСП показал, что эффективность МО преобразования увеличивается при этом на порядок. Более высокую эффективность преобразования можно достичь при использовании феррит-гранатовых пленок с анизотропией „легкая плоскость“ при визуализации тангенциальной компоненты магнитного потока, захваченного элементами матрицы ВТСП при охлаждении еe ниже $T_{\text {cr }}$ во внешнем магнитном поле. Это позволяет значительно повысить чувствительность матричного приемника ИК излучений за счет повышения рабочей температуры ВТСП матрицы и снижения напряженности внешнего подмагничивающего поля. Кроме того, использование тангенциальной компоненты дает возможность проводить измерения по компенсационной методике, поскольку дифференциальный метод позволяет существенно (на два порядка) снизить влияние флуктуаций оптического излучения на величину шумов измеряемого сигнала [5].

Следует заметить, что концентрационная способность периодической структуры в виде матрицы сверхпроводниковых чувствительных элементов дает возможность предложить устройство для регистрации малых магнитных полей - планарный магнитооптический магнитометр с чувствительностью $\sim 10^{-12} \mathrm{~T}$, который будет описан позже. 


\section{Список литературы}

[1] Есиков О.С., Протасов Е.А. // Опт. и спектр. 2011. Т. 110. № 6. C. 1021.

[2] Физические свойства высокотемпературных сверхпроводников / Под ред. Д.М. Гинзберга. М.: Мир, 1990.

[3] Пирогов Ю.Н., Протасов Е.А. // ЖТФ. 2000. Т. 70. Вып. 9. C. 128.

[4] Звездин А.К., Котов В.А. Магнитооптика тонких пленок. М.: Наука, 1988.

[5] Лебедев С.В. // ЖТФ. 1993. Т. 63. Вып. 11. С. 72. 